\title{
Coupled Analysis of Microstructure Evolution with Creep Deformation in Nickel-Based Superalloys by the Phase-Field Method
}

\author{
Yuhki Tsukada $^{1}$, Yoshinori Murata ${ }^{2}$, Toshiyuki Koyama ${ }^{1}$, Nobuhiro Miura ${ }^{3}$, Yoshihiro Kondo ${ }^{3}$ \\ ${ }^{1}$ Department of Materials Science and Engineering, Graduate School of Engineering, Nagoya Institute of Technology, \\ Gokiso-cho, Showa-ku, Nagoya 466-8555, Japan \\ ${ }^{2}$ Department of Materials, Physics and Energy Engineering, Graduate School of Engineering, Nagoya University, \\ Furo-cho, Chikusa-ku, Nagoya 464-8603, Japan \\ ${ }^{3}$ Department of Mechanical Systems Engineering, School of Systems Engineering, National Defense Academy, \\ 1-10-20 Hashirimizu, Yokosuka 239-8686, Japan
}

Keywords: Phase-field method; Plasticity; Creep; Directional coarsening; CMSX-4

\begin{abstract}
Directional coarsening of the $\gamma^{\prime}$ phase (rafting) during creep in nickel-based superalloys was simulated by a phase-field model that calculates the evolution of both the $\left(\gamma+\gamma^{\prime}\right)$ microstructure and the inelastic strain in the $\gamma$ phase. A two-dimensional simulation revealed that directional coarsening affected the macroscopic creep behavior and that the strain rate increased temporarily. The creep rate-time curve of the initial stage of transient creep was successfully reproduced by the simulation. Furthermore, the results of a three-dimensional simulation showed that the dislocations prefered the $\gamma$ phase perpendicular to the external tensile stress and demonstrated that the strain rate increased locally at the $\gamma / \gamma$ ' interface with a curvature when rafting occurs.
\end{abstract}

\section{Introduction}

Nickel-based single-crystal superalloys exhibit superior creep strength at high temperatures. This excellent mechanical property originates from the cuboidal $\gamma^{\prime}$ strengthening phase $\left(\mathrm{L}_{2}\right.$ crystal structure) precipitated coherently in the $\gamma$ phase (A1 crystal structure) [1]. During high-temperature creep, the $\gamma^{\prime}$ phase undergoes a directional coarsening; if the lattice misfit is negative $\left(a_{\gamma}>a_{\gamma^{\prime}}\right), \gamma^{\prime}$ particles coarsen toward the direction perpendicular to the [001] tensile-stress axis and connect with each other [2]. The resultant microstructure is often called a rafted structure. The examination of the microstructure evolution in CMSX-4 during creep at $1273 \mathrm{~K}$ under low stress [3] has revealed that the $\gamma$ channel width of the rafted structure increases as a result of the coalescence of the $\gamma^{\prime}$ phase. The examination has also revealed that the creep rate at the accelerating creep stage is closely related to the increase in the $\gamma$-channel width, namely, the microstructure evolution [3]. On the other hand, some phase-field simulations have clarified that the rafting phenomenon is driven mainly by plasticity in the $\gamma$ phase [4-7]. Hence, microstructure evolution and creep deformation are correlated, and the details of the correlation need to be understood quantitatively to predict the creep strength of superalloys.

Recently, a phase-field model that treats the evolution of both the $(\gamma+\gamma)$ microstructure and the plasticity in the $\gamma$ phase simultaneously was developed [8]. In this model, it is possible to simulate the microstructure evolution driven by the gradient of the elastic potential field associated with both inelastic strain in the $\gamma$ phase and coherent strain between the $\gamma$ and $\gamma$ ' phases. The evolution of creep strain is calculated on the basis of the von Mises-type creep theory by incorporating the macroscopic creep behavior previously reported for a single crystal of a $\gamma$-phase alloy. In this study, the microstructure evolution during creep in CMSX4 is simulated using the above mentioned phase-field model. Results of a two-dimensional (2D) simulation are compared with previous experimental data on the creep rate-time curve and the corresponding microstructure evolution. In addition, a part of the results from a three-dimensional (3D) simulation, the analysis of which is still ongoing, is also shown.

\section{Calculation method}

Phase-field model

To describe the $\left(\gamma+\gamma^{\prime}\right)$ microstructure, the volume fraction field of the $\gamma^{\prime}$ phase, $f(\boldsymbol{r}, t)$, and four structural-order parameter fields distinguishing four different ordered domains, $\phi_{i}(\boldsymbol{r}, t), i=1,2,3,4$, are employed. These field variables are functions of space $(\boldsymbol{r})$ and time $(t)$, and change continuously across the $\gamma / \gamma^{\prime}$ interfacial regions. In this study, it is assumed that $f(r, t)$ is related to the composition field, $c(\boldsymbol{r}, t)$, as

$$
f(\boldsymbol{r}, t)=\frac{c(\boldsymbol{r}, t)-c_{\gamma}^{0}}{c_{\gamma^{\prime}}^{0}-c_{\gamma}^{0}},
$$

where $c^{0}{ }_{\gamma}$ and $c^{0}{ }^{\gamma}$ are the equilibrium compositions of the $\gamma$ and $\gamma$, phases, respectively. The temporal evolution of the field variables is given by the following Cahn-Hilliard and Allen-Cahn equations [9]:

$$
\begin{aligned}
& \frac{\partial f(\boldsymbol{r}, t)}{\partial t}=M_{f} \nabla^{2} \frac{\delta G}{\delta f(\boldsymbol{r}, t)}, \\
& \frac{\partial \phi_{i}(\boldsymbol{r}, t)}{\partial t}=-L \frac{\delta G}{\delta \phi_{i}(\boldsymbol{r}, t)}, i=1,2,3,4 .
\end{aligned}
$$

Here, $G$ is the total free energy of the microstructure, $M_{f}$ is the interfacial mobility, and $L$ is the structural relaxation coefficient. In this study, both $M_{f}$ and $L$ are assumed to be constants.

The total free energy is given by 


$$
G=\int_{r}\left[\begin{array}{l}
\left\{1-h\left(\phi_{i}\right)\right\} G_{\text {chem }}^{\gamma}\left(f_{\gamma}\right)+h\left(\phi_{i}\right) G_{\text {chem }}^{\gamma^{\prime}}\left(f_{\gamma}\right) \\
+w g\left(\phi_{i}\right)+\frac{\kappa_{\phi}}{2}\left(\nabla \phi_{i}\right)^{2}
\end{array}\right] d \boldsymbol{r}+E_{\text {str }},
$$

where $G_{\text {chem }}^{\gamma}$ and $G^{\gamma^{\prime}}$ chem are the chemical free energy densities of the $\gamma$ and $\gamma^{\prime}$ phases, respectively; $\kappa_{\phi}$ is the gradient energy coefficient [10]; and $E_{\mathrm{str}}$ is the elastic strain energy. The $\gamma / \gamma$ ' interfacial region is regarded as a mixture of $f_{\gamma}$ and $f_{\gamma}$, which have different $\gamma^{\prime}$ volume fractions but equal chemical potentials $[8,11]$. $h\left(\phi_{i}\right)$ is a continuous function with values between 0 and $1, g\left(\phi_{i}\right)$ is the double-well potential, and $w$ is the double-well potential height. The functions $h\left(\phi_{i}\right)$ and $g\left(\phi_{i}\right)$ are chosen as [12]

$$
\begin{aligned}
& h\left(\phi_{i}\right)=\sum_{i=1}^{4}\left[\phi_{i}^{3}\left(10-15 \phi_{i}+6 \phi_{i}^{2}\right)\right], \\
& g\left(\phi_{i}\right)=\sum_{i=1}^{4}\left[\phi_{i}^{2}\left(1-\phi_{i}\right)^{2}\right]+\alpha \sum_{i=1}^{4} \sum_{j \neq i}^{4} \phi_{i}^{2} \phi_{j}^{2} .
\end{aligned}
$$

The parameters $w$ and $\kappa_{\phi}$ in Eq. (4) are related to both the $\gamma / \gamma^{\prime}$ interfacial energy per area and the thickness of the $\gamma / \gamma^{\prime}$ diffuse interface [12].

The chemical free energy densities of both phases are formulated as a function of composition and temperature, and can be calculated from the thermodynamic database of the equilibrium phase diagram. In this study, it is assumed that the free energy densities can be approximated as quadratic functions of $f(r, t)$ as

$$
\begin{aligned}
& G_{\text {chem }}^{\gamma}=W_{\gamma} f^{2}, \\
& G_{\text {chem }}^{\gamma^{\prime}}=W_{\gamma^{\prime}}(1-f)^{2},
\end{aligned}
$$

where $W_{\gamma}$ and $W_{\gamma^{\prime}}$ are the coefficients determined by fitting Eqs. (7) and (8) to the free energy curve of each phase.

The elastic strain energy is given as [13-15]

$$
E_{\mathrm{str}}=\int_{r}\left[\begin{array}{l}
\frac{1}{2} C_{i j k l}(\boldsymbol{r})\left\{\varepsilon_{i j}(\boldsymbol{r})-\varepsilon_{i j}^{0}(\boldsymbol{r})\right\}\left\{\varepsilon_{k l}(\boldsymbol{r})-\varepsilon_{k l}^{0}(\boldsymbol{r})\right\} \\
-\sigma_{i j}^{\mathrm{appl}} \bar{\varepsilon}_{i j}
\end{array}\right] d \boldsymbol{r},
$$

where $C_{i j k l}$ is the elastic constant, $\varepsilon$ is the total strain, $\varepsilon^{0}$ is the eigenstrain, $\sigma^{\text {appl }}$ is the applied stress, and $\bar{\varepsilon}$ is the uniform macroscopic strain. The total strain can be calculated from the local equilibrium equation once the spatial distribution of the eigenstrain is given. The eigenstrain is defined as

$\varepsilon_{i j}^{0}(\boldsymbol{r})=\varepsilon_{0} \delta_{i j} h\left(\phi_{i}\right)+\varepsilon_{i j}^{\mathrm{p}}(\boldsymbol{r})+\varepsilon_{i j}^{\mathrm{c}}(\boldsymbol{r})$,

where $\delta_{i j}$ is the Kronecker delta function. The first term on the right-hand side of Eq. (10) is the lattice misfit strain between the $\gamma$ and $\gamma^{\prime}$ phases, and $\varepsilon_{0}$ is the lattice misfit given by $\varepsilon_{0}=\left(a_{\gamma^{\prime}}-a_{\gamma}\right) / a_{\gamma}$, where $a_{\gamma}$ and $a_{\gamma}$, represent the lattice parameters of the $\gamma$ and $\gamma$ ' phases, respectively. The remaining two terms in the right-hand side of Eq. (10), $\varepsilon_{i j}^{p_{j}}$ and $\varepsilon_{i j}^{c}$, are the plastic strain and the creep strain, respectively. It is assumed on the basis of a previous report on the microstructure observation of a crept specimen [2] that both of these strains are confined in the $\gamma$ phase.

Evolution of plastic strain and creep strain

The plastic strain $\left(\varepsilon_{i j}^{p_{i j}}\right)$ is defined as time-independent inelastic strain. The evolution of $\varepsilon_{i j}$ is given by [16]

$$
\frac{\partial \varepsilon_{i j}^{\mathrm{p}}\left(\boldsymbol{r}_{\mathrm{p}}, \tau\right)}{\partial \tau}=-K_{i j k l} \frac{\delta E_{\text {shear }}}{\delta \varepsilon_{k l}^{\mathrm{p}}\left(\boldsymbol{r}_{\mathrm{p}}, \tau\right)},
$$

where $\tau$ is dimensionless time, $E_{\text {shear }}$ is shear strain energy, and $K_{i j k l}$ is the kinetic coefficient. $E_{\text {shear }}$ is given by

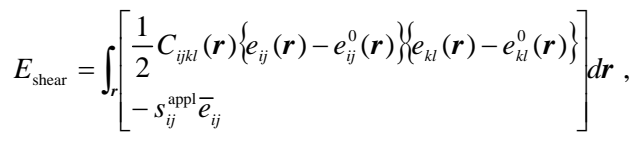

where $e_{i j}$ and $s_{i j}$ are the deviatoric strain and stress, respectively. Eq. (11) is solved at the location $\left(\boldsymbol{r}_{\mathrm{p}}\right)$ where the von Mises yield criterion is exceeded, and the elastic-perfectly plastic relation is assumed for simplicity.

The creep strain $\left(\varepsilon_{i j}\right)$ is defined as time-dependent inelastic strain. On the basis of the von Mises-type creep theory [17], the evolution of $\varepsilon_{i j}^{c}$ is given by

$\frac{\partial \varepsilon_{i j}^{c}(\boldsymbol{r}, t)}{\partial t}=\frac{3}{2} C \bar{\sigma}^{4} s_{i j}(\boldsymbol{r}, t)$,

where $C$ is a material constant and $\bar{\sigma}$ is the equivalent stress. In Eq. (13), the creep property of the single crystal of a $\gamma$-phase alloy is incorporated so that the five-power law behavior is observed in the early stage of transient creep $[18,19]$.

\section{Calculation results}

2D simulation

A two-dimensional (2D) simulation is performed by solving Eqs. (2) and (3) numerically by the difference method, and the periodic boundary conditions are assumed along two dimensions. In numerical analysis, all the physical parameters are reduced to dimensionless quantities. In particular, the time scale is reduced to dimensionless time using the interfacial mobility, $M_{f}$, as $t^{*}=t \times$ $\left(M_{f} R T / l^{2}\right)$. Here, the asterisk denotes a dimensionless quantity in numerical analysis, $R$ is the gas constant, $T$ is the absolute temperature, and $l$ is the unit grid size.

The parameters used in the simulation are listed in Table I. Physical parameters of CMSX-4, the elastic constants and the lattice misfit, are employed $[20,21]$. The local elastic constant is assumed as $C_{i j k l}(\boldsymbol{r})=\left\{1-h\left(\phi_{i}\right)\right\} C^{\gamma}{ }_{i j k l}+h\left(\phi_{i}\right) C^{\gamma_{i j k l}^{\prime}}$, where $C^{\gamma}{ }_{i j k l}$ and $C^{\gamma^{\prime}{ }_{i j k l}}$ represent the elastic constants of the $\gamma$ and $\gamma^{\prime}$ phases, respectively. The structural relaxation coefficient in Eq. (3) is set as $L=5 M_{f}$ to ensure that the process of microstructure evolution is diffusion controlled. The kinetic coefficient in Eq. (11) is assumed as $K_{i j k l}=K \delta_{i k} \delta_{j l}$, and $K$ is set as $K^{*}=0.002$. The material constant, $C$, in Eq. (13) is set as $C^{*}=0.3$; this constant is a fitting parameter to reproduce the creep rate-time curve obtained in the previous experiment. 
Table I. Parameters used in the 2D simulation.

\begin{tabular}{|c|c|}
\hline Computational cell & $32 \times 32$ \\
\hline System size, $D \times D\left(\mathrm{~nm}^{2}\right)$ & $256 \times 256$ \\
\hline Temperature, $T$ (K) & 1273 \\
\hline External tensile stress, $\sigma^{\text {appl }}(\mathrm{MPa})$ & 160 \\
\hline Free energy coefficients, $W\left(\mathrm{~J} \mathrm{~m}^{-3}\right)$ & $W_{\gamma}=1.29 \times 10^{8}$ \\
\hline$[8]$ & $W_{\gamma^{\prime}}=1.56 \times 10^{8}$ \\
\hline $\begin{array}{l}\text { Double-well potential height, } \\
w\left(\mathrm{~J} \mathrm{~m}^{-3}\right)[8]\end{array}$ & $1.07 \times 10^{7}$ \\
\hline $\begin{array}{l}\text { Gradient energy coefficient, } \\
\kappa_{\phi}\left(\mathrm{J} \mathrm{m}^{-1}\right)[8]\end{array}$ & $3.41 \times 10^{-10}$ \\
\hline $\begin{array}{l}\text { Average volume fraction of the } \\
\gamma^{\prime} \text { phase in the system }\end{array}$ & 0.66 \\
\hline Elastic constants, $C_{i j k l}(\mathrm{GPa})[20]$ & $\begin{array}{l}C^{\gamma}{ }_{11}=204.9, C^{\gamma^{\prime}}{ }_{11}=204.9 \\
C^{\gamma_{12}}=150.8, C^{\gamma^{\prime}}=194.5 \\
C^{\gamma}{ }_{44}=94.0, C_{44}^{\gamma^{\prime}}=95.0\end{array}$ \\
\hline Lattice misfit, $\varepsilon_{0}[21]$ & -0.0023 \\
\hline $\begin{array}{l}\text { Yield stress of the } \gamma \text { phase, } \\
\sigma_{\mathrm{y}}(\mathrm{MPa})[8]\end{array}$ & 120 \\
\hline Time step, $\Delta t^{*}$ & 0.2 \\
\hline Time step, $\Delta \tau$ & 0.0002 \\
\hline
\end{tabular}

Figure 1 shows the initial microstructure of the simulation. The black and white areas correspond to the $\gamma$ and $\gamma^{\prime}$ phases, respectively. The square $\gamma$ ' phase with an edge length of about $200 \mathrm{~nm}$ is placed at the center of the computational cell. As mentioned previously, a periodic boundary condition is assumed. Hence, the initial state can be regarded as a periodic array of the $\gamma$ ' phase along the $<10>$ crystallographic directions.

Figure 2 shows the spatial distribution of plastic strain immediately after the loading of external tensile stress along the [01] direction $\left(t^{*}=0\right)$. Plastic strain has been introduced, so the equivalent stress is less than the yield stress in the entire $\gamma$-phase region. At this moment, creep strain has not yet been introduced. It is seen that the plastic strain concentrates at the $\gamma$ phase along the [10] direction. This result is qualitatively consistent with a previous analysis [4] that dislocations prefer the $\gamma$ channels perpendicular to the [001] tensile-stress axis.

The time evolution of the microstructure is shown in Fig. 3 (a), and the corresponding spatial distributions of the inelastic strain and the inelastic-strain rate are shown in Figs. 3 (b) and (c), respectively. Here, the inelastic strain is given by the sum of the plastic strain and the creep strain. It is seen from Fig. 3 (a) that the square $\gamma$ ' phase gradually evolves toward the [10] crystallographic direction $\left(t^{*}=6,000\right)$ and connects with neighboring $\gamma^{\prime}$ particles $\left(t^{*}=14,400\right)$. Finally, directional coarsening leads to the formation of a rafted structure $\left(t^{*}=40,000\right)$. During the microstructure evolution, creep strain is introduced in the $\gamma$ phase according to Eq. (13) and increases with time. As shown in Fig. 3 (b), when the morphology of the square $\gamma^{\prime}$ phase is maintained ( $t^{*}$

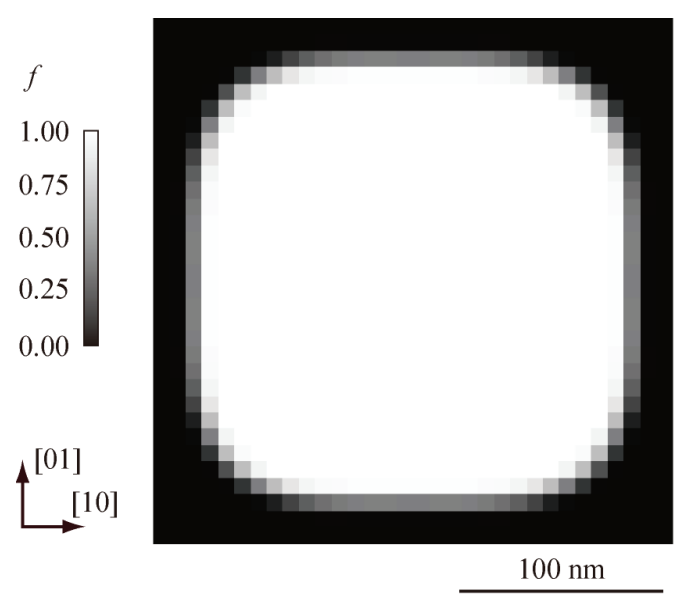

Figure 1. Initial microstructure in 2D simulation. Black and white areas represent the $\gamma$ and $\gamma$ ' phases, respectively.

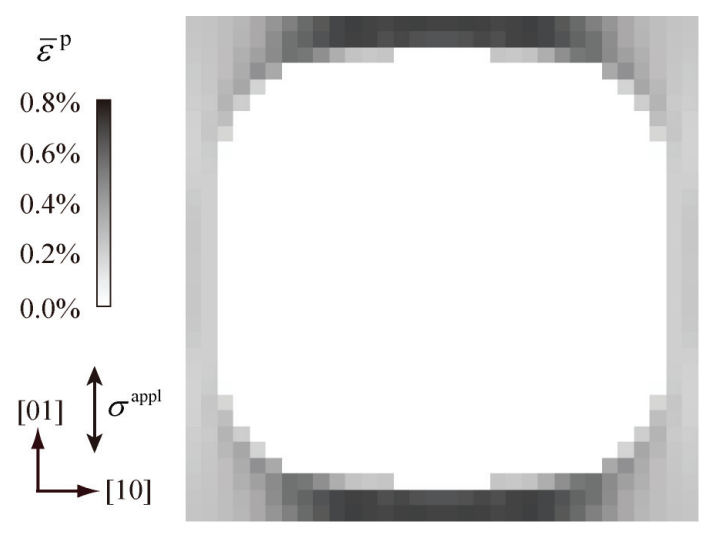

Figure 2. Spatial distribution of the equivalent plastic strain immediately after the loading of external tensile stress along the [01] crystallographic direction.

$=6,000)$, a large amount of inelastic strain is observed preferentially in the $\gamma$ phase along the [10] crystallographic direction. However, as the rafted structure is formed $\left(t^{*}=\right.$ $14,400-40,000)$, the inelastic strain region is gradually enlarged in the entire $\gamma$ phase.

Fig. 3 (c) shows that the strain rate is high at the $\gamma / \gamma^{\prime}$ interface with a curvature $\left(t^{*}=6,000\right)$. Furthermore, it should be noted that the strain rate at the interface prominently increases when the $\gamma^{\prime}$ particles connect with each other through directional coarsening 


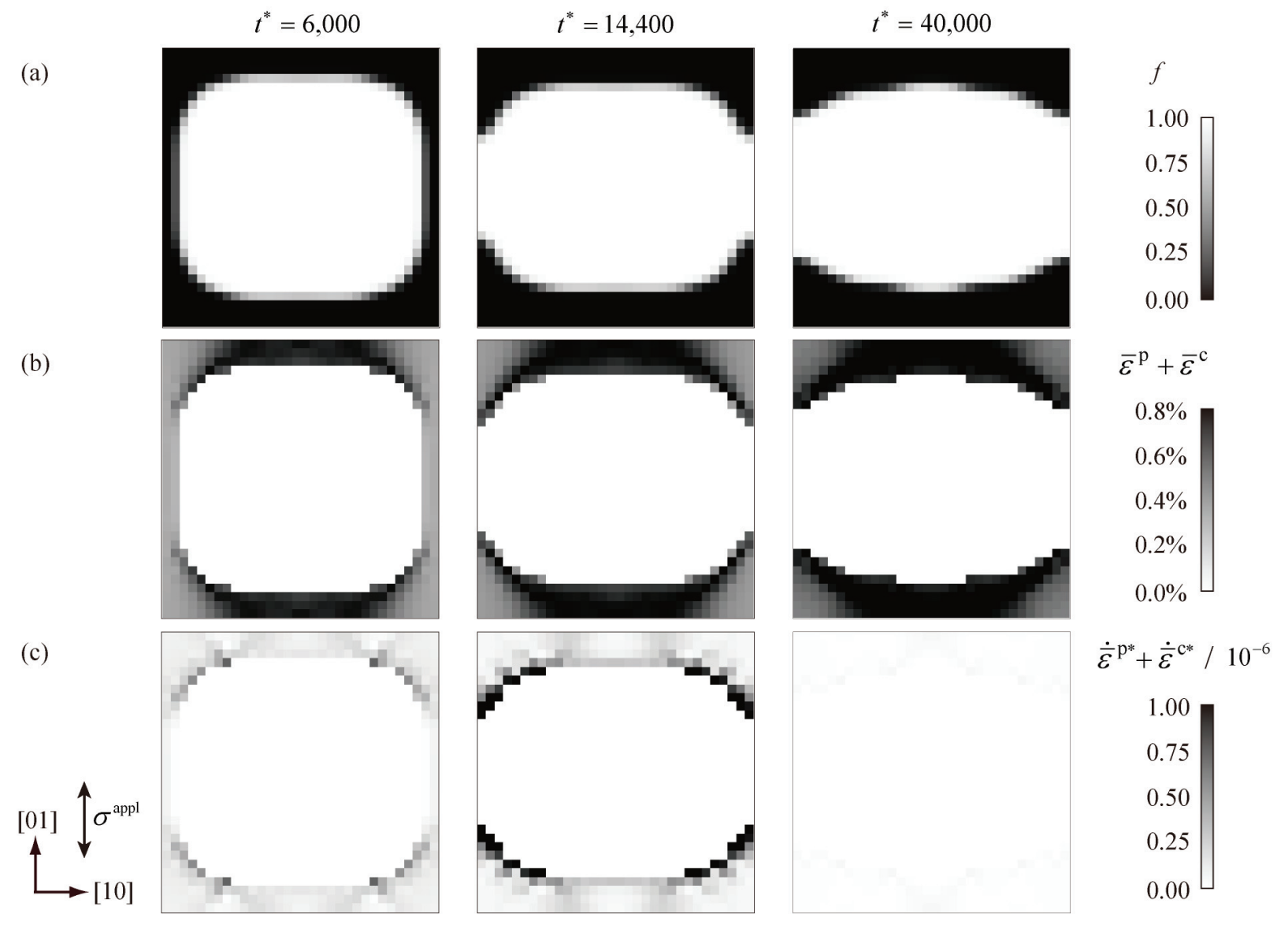

Figure 3. Result of 2D phase-field simulation: (a) morphological evolution of the $\gamma^{\prime}$ phase, (b) spatial distribution of the equivalent inelastic strain, and (c) spatial distribution of the equivalent inelastic-strain rate. The inelastic strain is the sum of the plastic strain $\left(\varepsilon^{p_{i j}}\right)$ and the creep strain $\left(\varepsilon^{c}\right)$.

$\left(t^{*}=14,400\right)$. Finally, after the rafted structure forms, the strain rate continues to decrease to a negligible amount $\left(t^{*}=40,000\right)$. As is understood from Eq. (13), the local inelastic-strain rate in the $\gamma$ phase is correlated with the distribution of internal stress; the higher the local stress state, the higher is the local strain rate. Hence, the simulation result shown in Fig. 3 (c) at $t^{*}=14,400$ assumes that the equivalent stress at the $\gamma / \gamma^{\prime}$ interface is high temporarily when vertical $\gamma$ channels start to disappear.

The creep rate-time curve obtained by the simulation is shown by solid symbols in Fig. 4. From the beginning of creep, a monotonic decrease in the creep rate is observed. The creep rate temporarily increases around $t^{*}=14,400$, when the rafted structure forms. After $t^{*}=14,400$, the creep rate continues to decrease, and the accelerating creep stage is not reproduced in the simulation. It has been reported that the temporary increase in the macroscopic strain rate is observed experimentally in the early stage of hightemperature creep under low stress [22]. From Fig. 3 (c), it is assumed that the stress concentration at the $\gamma / \gamma^{\prime}$ interface is the direct cause of the temporary increase in the macroscopic creep rate. The simulation results in this study reveal the correlation between directional coarsening during creep and macroscopic creep response.

In Fig. 4, the creep rate-time curve of CMSX-4 under an external tensile stress of $160 \mathrm{MPa}$ along the [001] crystallographic direction is shown by open symbols [3]. In this condition, the rafted structure forms before the minimum creep rate, $t=200 \mathrm{~h}$, is 


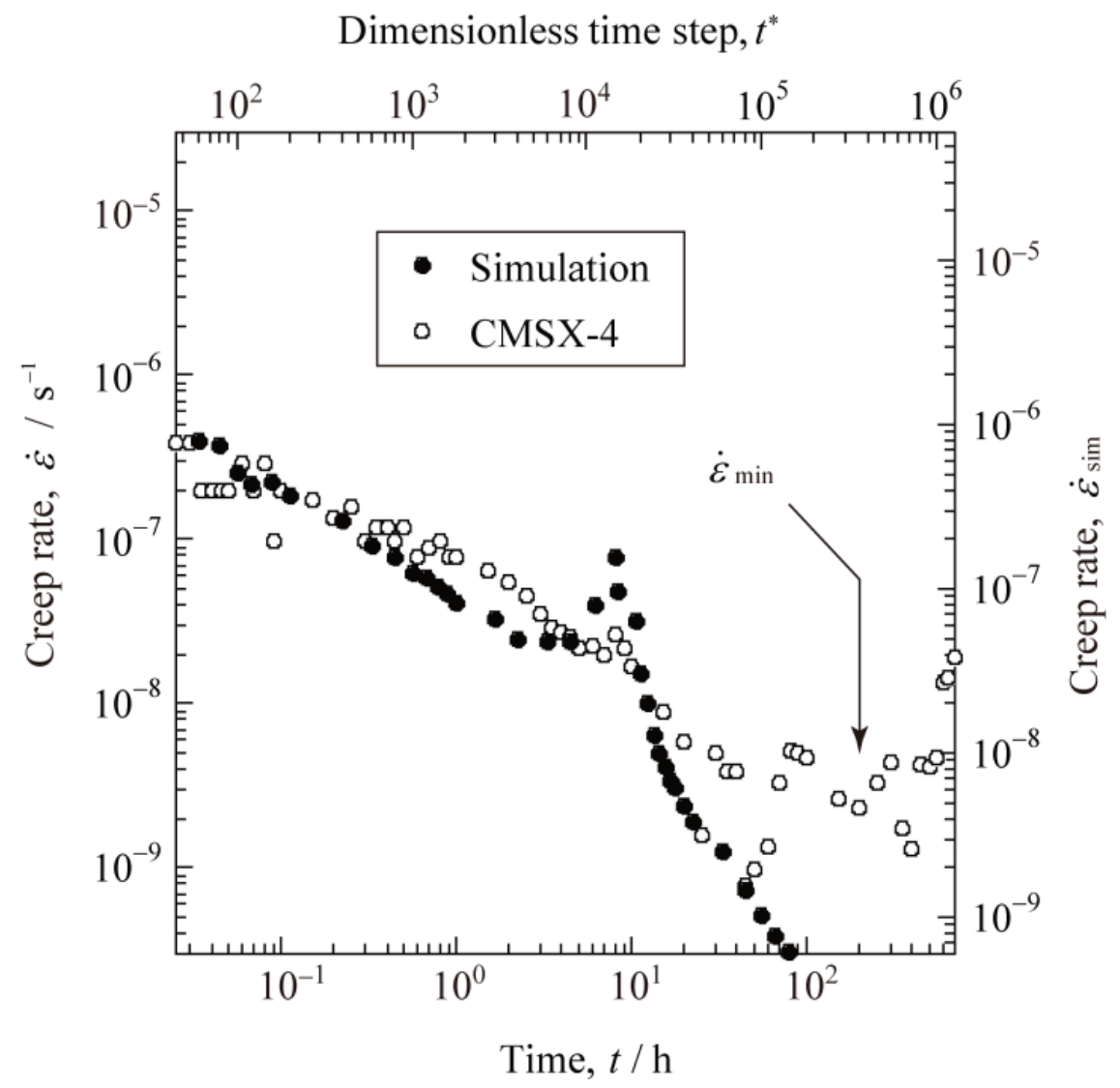

Figure 4. Creep rate-time curves obtained from the 2D simulation (solid symbols) and from the experiment on CMSX-4 (open symbols) [3]. The arrow indicates the time at which the minimum creep rate is reached in the experiment.

attained. In fact, directional coarsening has already started at $t=$ $30 \mathrm{~h}$ as shown in Fig. 5; as indicated by arrows in the SEM micrograph, it is observed that the rafted structure is partially formed. Hence, it is assumed that the increase in the creep rate at $t$ $=8 \mathrm{~h}$ originates from directional coarsening. In Fig. 4, simulation time steps are transformed into real time values by assuming that $t^{*}=14,400$ corresponds to $t=8 \mathrm{~h}$. As mentioned earlier, the correlation of simulation time step with real time value is described by $t^{*}=t \times\left(M_{f} R T / l^{2}\right)$. Hence, the interfacial mobility is calculated as $M_{f}=3.02 \times 10^{-21} \mathrm{~J}^{-1} \mathrm{~mol} \mathrm{~m} \mathrm{~s}^{-1}$, which is consistent well with the value approximated from the diffusion mobility database [23]. It is seen from Fig. 4 that the experimental curve in the early stage of the transient creep is successfully reproduced by the simulation. However, there is a large discrepancy between the simulated and experimental curves in the later stage of creep.

3D simulation

Three-dimensional (3D) simulation is performed to confirm the reproducibility of directional coarsening in 3D analysis. The computational cell is $32 \times 32 \times 32$, and the system size is $256 \times$ $256 \times 256 \mathrm{~nm}^{3}$. The average volume fraction of the $\gamma$ ' phase in the system is set as 0.54 . The material constant in Eq. (13) is set as $C^{*}$ $=0.02$. Other parameters employed for the simulation are the same as those listed in Table I. Figure 6 shows the initial microstructure of the simulation. The cuboidal $\gamma^{\prime}$ phase, whose edge length is about $200 \mathrm{~nm}$, is placed at the center of the computational cell. The periodic boundary conditions are assumed along all three dimensions.

Figure 7 (a) shows the 3D microstructure evolution obtained by the simulation. The corresponding 3D contours of the inelastic strain and the inelastic-strain rate are shown in Figs. 7 (b) and (c), respectively. An external tensile stress of $160 \mathrm{MPa}$ is applied along the [001] crystallographic direction. It is observed in Fig. 7 (a) that the $\gamma$ ' phase evolves toward the direction perpendicular to the external tensile stress $\left(t^{*}=50,000\right)$, resulting in a connection with neighboring $\gamma^{\prime}$ particles $\left(t^{*}=95,000\right)$. Calculation of the subsequent microstructure evolution is currently under way, but it 


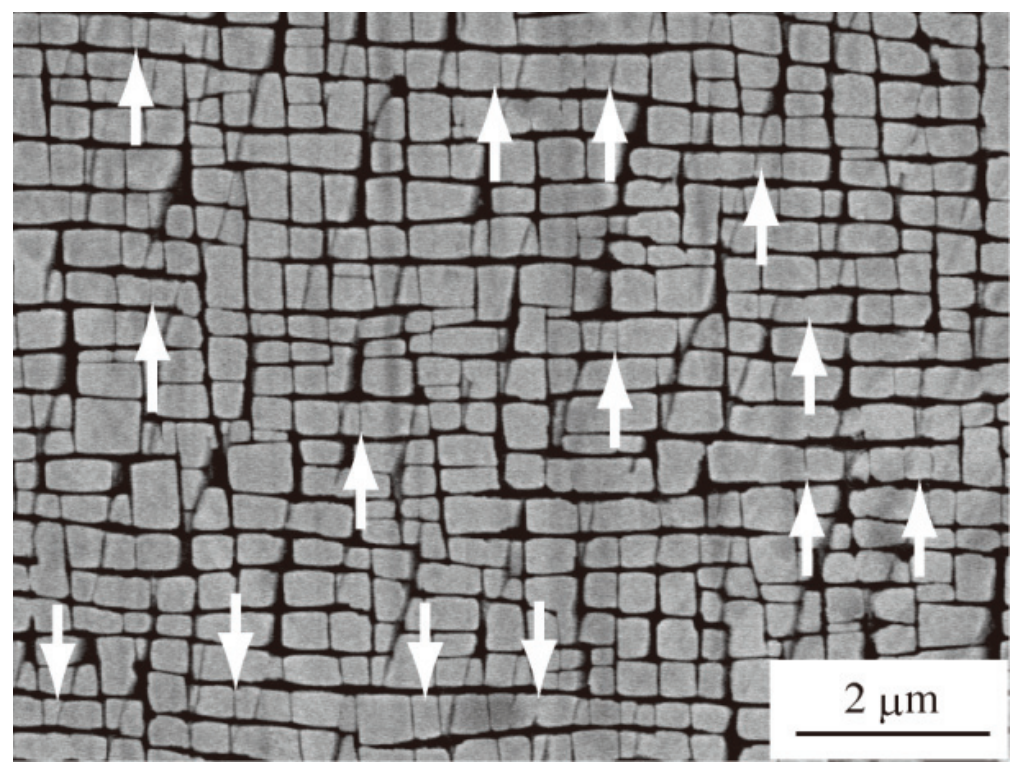

Figure 5. SEM micrograph of CMSX-4 crept at $1273 \mathrm{~K}$ under $160 \mathrm{MPa}$ for $30 \mathrm{~h}$.

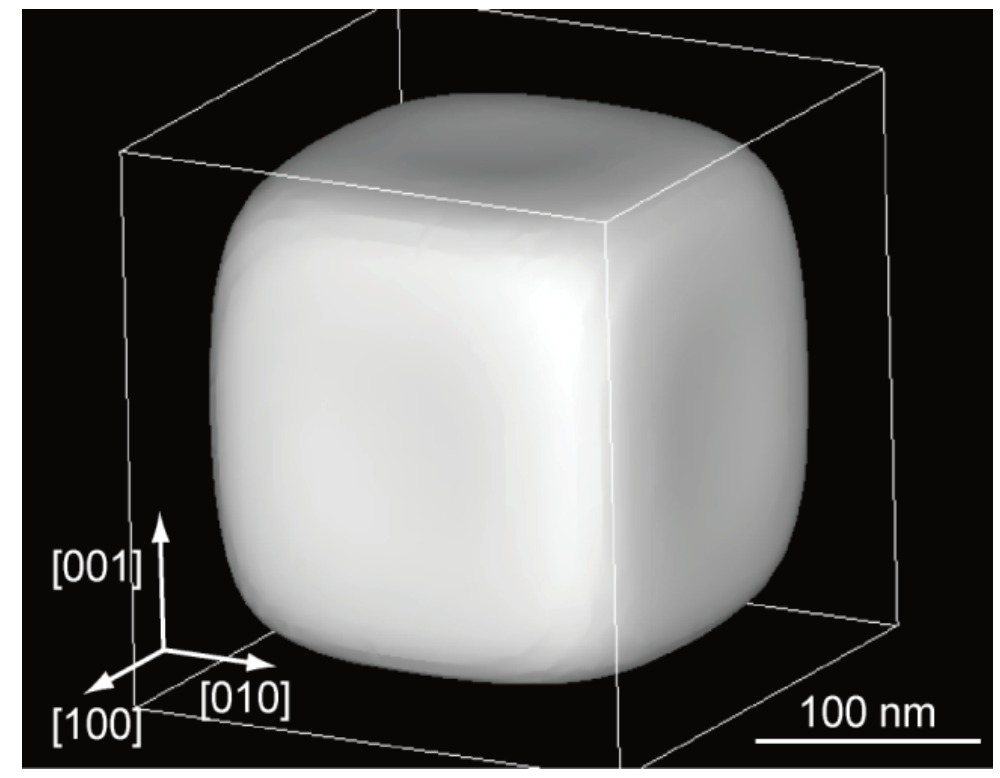

Figure 6. Initial microstructure in 3D simulation. The cuboidal $\gamma$ ' phase is placed at the center of the computational cell. 
$t^{*}=50,000$

(a)

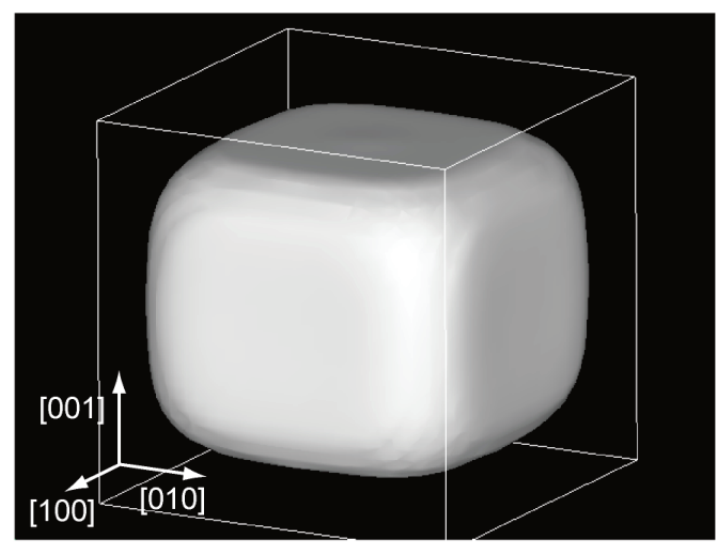

(b)

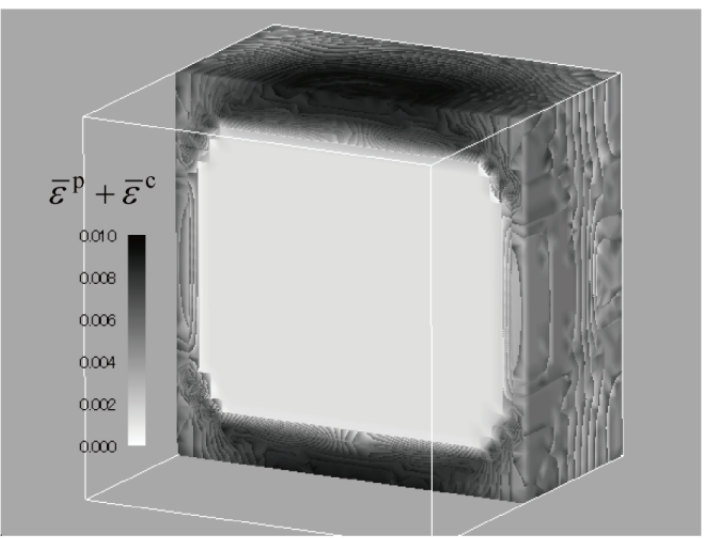

(c)

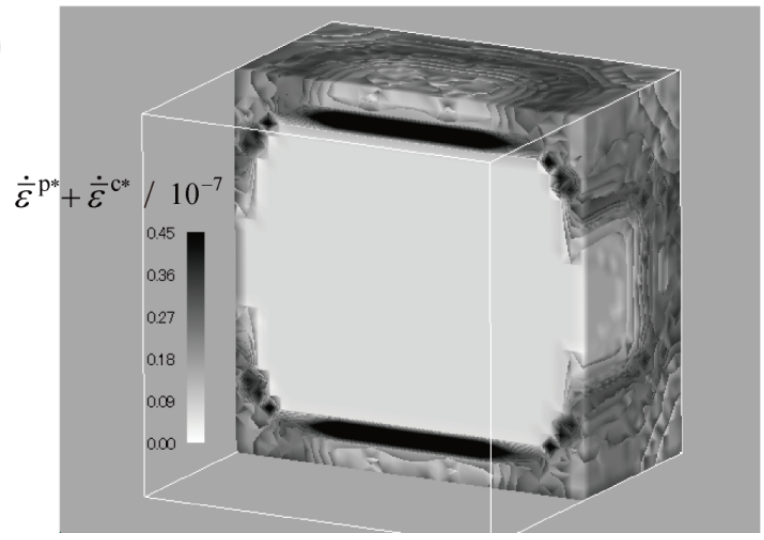

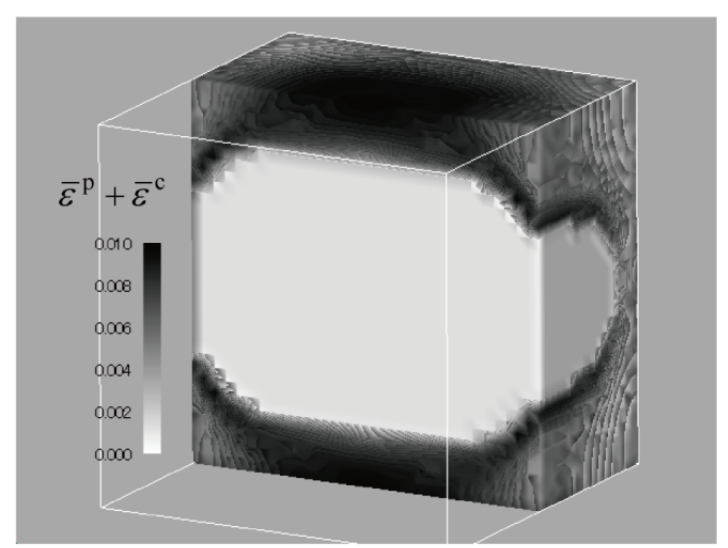

$t^{*}=95,000$
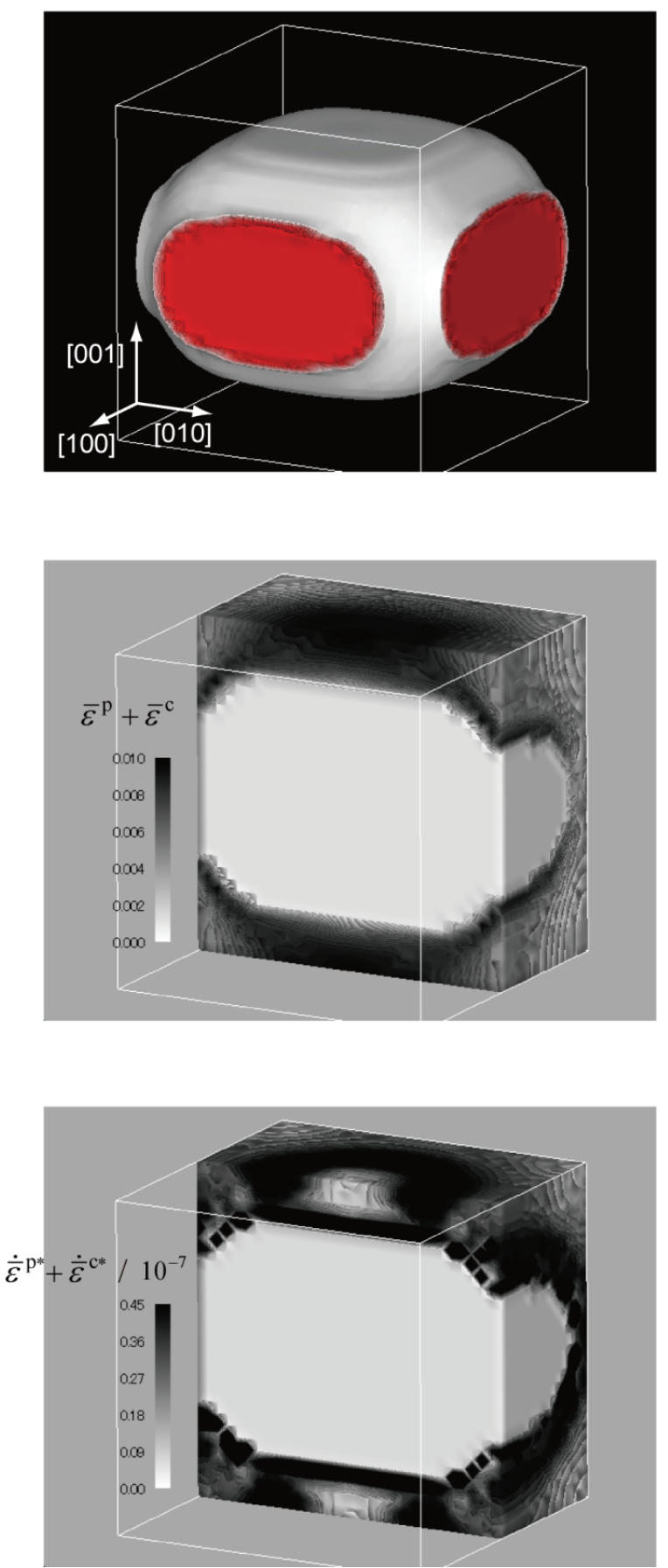

Figure 7. 3D simulation results of (a) microstructure evolution, (b) 3D contour of the inelastic strain, and (c) 3D contour of the inelastic-strain rate. An external tensile stress of $160 \mathrm{MPa}$ is applied along the [001] crystallographic direction. 
is expected that an ideal (001) rafted structure is formed. It is worth noting from Fig. 7 (b) that the inelastic strain is concentrated at the (001) $\gamma$ channel. Furthermore, when the $\gamma$ ' phase forms the (001) rafted structure $\left(t^{*}=95,000\right)$, the inelasticstrain rate is locally high not only at the $\gamma / \gamma^{\prime}$ interface with a curvature but also at a part of the (001) $\gamma$ channel (see Fig. 7 (c)). The temporary increase in the strain rate could affect the macroscopic creep behavior as shown in Fig. 4 for the 2D simulation. The creep rate-time curve based on the 3D simulation, which will be obtained by our ongoing numerical analysis, is required to reveal the detail of the microstructure evolution and its resulting macroscopic creep response.

\section{Discussion}

Discrepancy between the simulation and experimental results As shown in Fig. 4, the creep rate-time curve of CMSX-4 in the late stage of creep has not been reproduced. During long-term creep at high temperatures in CMSX-4, microstructure evolution after formation of the rafted structure has been observed; the coalescence of the $\gamma$ ' phase broadens the width of the $\gamma$-phase channels [3]. It has been revealed that this microstructure change leads to an increase in the strain rate. In the numerical simulation in this study, an ideal array of the $\gamma^{\prime}$ phase is assumed as the initial microstructure. Hence, directional coarsening of the $\gamma^{\prime}$ phase forms an ideal lamellar structure. As a result, the coalescence of the $\gamma^{\prime}$ phase cannot be reproduced by the simulation; this is the direct cause of the discrepancy between the simulation and experimental results. A large-scale simulation incorporating spatial inhomogeneity of $\gamma$ ' particles could reproduce the coalescence of the rafted structure. The simulation results in this study indicate that the high-temperature creep strength of superalloys can be dramatically improved if the ideal lamellar structure is maintained.

\section{Modeling method of inelastic strain}

In the phase-field model of dislocations [24], the spatial distribution of inelastic strain associated with an arbitrary dislocation configuration is described through the product of the eigenstrain of a dislocation loop and the order parameter that serves as a shape function of the slipped region. In this model, the total energy of a crystal with dislocations is described as a function of the order parameter, whose time evolution is calculated as the steepest descent path of the total energy. Hence, dislocation reactions such as multiplication and annihilation of dislocations can be taken into account. However, it is difficult to treat quantitatively the motion of dislocation climb, which is an important thermal activation process in high-temperature creep.

On the other hand, in this study, the inelastic strain field in the $\gamma$ phase is defined as the sum of plastic strain $\left(\varepsilon^{\mathrm{p}}\right)$ and creep strain $\left(\varepsilon^{c}\right)$. The evolution of inelastic strain is calculated on the basis of flow rule, in which the principle of maximum plastic work is assumed. This modeling method enables one to incorporate directly the previously reported macroscopic creep properties of $\gamma$ phase alloys. The adoption of this model has led to the reproduction of both the microstructure evolution and the creep rate-time curve in the initial stage of transient creep, as shown in Figs. 3 and 4, respectively. However, in contrast to the phase-field model of dislocations, solving Eq. (11) or (13) does not automatically lead to a minimum-energy inelastic strain configuration.
In high-temperature creep, the dislocation networks form at the $\gamma / \gamma^{\prime}$ interface. It has been reported that as the interfacial dislocation spacing becomes smaller, the creep rate lessens [25]. Consequently, in the near future, the method of modeling the evolution of the inelastic strain field, including dislocation climb, seems to be one of the most important issues for simulations based on the phase-field method.

\section{$\gamma / \gamma^{\prime}$ interfacial mobility}

The evolution of the $\gamma^{\prime}$ volume fraction field is solved, and the $\gamma / \gamma^{\text {' }}$ interfacial mobility is estimated as $M_{f}=3.02 \times 10^{-21} \mathrm{~J}^{-1} \mathrm{~mol} \mathrm{~m}{ }^{2}$ $\mathrm{s}^{-1}$ from the creep rate-time curve obtained by the 2D simulation. Our recent analysis [23] suggests that the estimated $\gamma / \gamma$ ' interfacial mobility exhibits good agreement with the value approximated from the diffusion mobility of rhenium (Re) in CMSX-4. This indicates that microstructure evolution in CMSX-4 is controlled by the atomic diffusion of $\mathrm{Re}$, which is one of the creepstrengthening elements.

If the creep rate-time curve is obtained by the 3D simulation, the interfacial mobility would be estimated more accurately; this could reveal a correlation between the interfacial mobility and atomic diffusion mobility, and in the near future, would enable the quantitative prediction of the $(\gamma+\gamma)$ microstructure evolution. The 3D simulation is ongoing, and details of the results will be presented in our forthcoming article.

\section{Conclusions}

The evolution of the $\left(\gamma+\gamma^{\prime}\right)$ microstructure during creep was simulated with a phase-field model that treated the evolutions of both the microstructure and the $\gamma$ phase plasticity simultaneously. Both the 2D and 3D simulations reproduced directional coarsening of the $\gamma^{\prime}$ phase and demonstrated the formation of a rafted structure. In the initial stage of creep, inelastic strain is concentrated preferentially in the $\gamma$ phase along the direction perpendicular to the external tensile-stress axis. When the $\gamma$ ' particles connect with each other through directional coarsening, the strain rate is increased locally at the $\gamma / \gamma$ ' interface with a curvature; the $2 \mathrm{D}$ analysis suggests that this phenomenon causes the increase in the macroscopic creep rate.

The creep rate-time curve in the initial stage of transient creep was successfully reproduced by the 2D simulation. However, there was a large discrepancy between the simulation and experimental results in the late stage of creep. For reproducing accelerating creep, the coalescence of the rafted structure needs to be simulated; hence, a large-scale simulation is required for considering the inhomogeneous alignment of $\gamma^{\prime}$ particles. Finally, the $\gamma / \gamma^{\prime}$ interfacial mobility should be estimated accurately from the creep rate-time curve obtained by the 3D simulation; this would enable one to understand the correlation between interfacial mobility and atomic diffusion mobility.

\section{Acknowledgment}

This work was supported by a Grant-in-Aid for JSPS Fellows of the Ministry of Education, Culture, Sports, Science and Technology (MEXT), Japan. 


\section{References}

1. R. C. Reed, The Superalloys Fundamentals and Applications (New York: Cambridge University Press, 2006), 33-120.

2. T. M. Pollock and A. S. Argon, "Directional Coarsening in Nickel-Base Single Crystals with High Volume Fractions of Coherent Precipitates,” Acta Metall Mater, 42 (1994), 1859-1874.

3. N. Miura, Y. Kondo, and T. Matsuo, "Relation between Creep Rate during Accelerating Creep Stage and $\gamma$ Channel Thickness in Single Crystal Nickel-Based Superalloy, CMSX-4," Tetsu-toHagané, 89 (2003), 1240-1247.

4. N. Zhou, C. Shen, M. J. Mills, and Y. Wang, "Phase Field Modeling of Channel Dislocation Activity and $\gamma$ ' Rafting in Single Crystal Ni-Al,” Acta Mater, 55 (2007), 5369-5381.

5. N. Zhou, C. Shen, M. J. Mills, and Y. Wang, "Contributions from Elastic Inhomogeneity and from Plasticity to $\gamma$ ' Rafting in Single-Crystal Ni-Al,” Acta Mater, 56 (2008), 6156-6173.

6. A. Gaubert, Y. L. Bouar, and A. Finel, "Coupling Phase Field and Viscoplasticity to Study Rafting in Ni-Based Superalloys," Philos Mag, 90 (2010), 375-404.

7. N. Zhou, C. Shen, M. Mills, and Y. Wang, "Large-Scale ThreeDimensional Phase Field Simulation of $\gamma$ '-Rafting and Creep Deformation,” Philos Mag, 90 (2010), 405-436.

8. Y. Tsukada, Y. Murata, T. Koyama, N. Miura, and Y. Kondo, "Creep Deformation and Rafting in Nickel-Based Superalloys Simulated by the Phase-Field Method Using Classical Flow and Creep Theories,” Acta Mater, 59 (2011), 6378-6386.

9. L. Q. Chen, "Phase-Field Models for Microstructure Evolution," Annu Rev Mater Res, 32 (2002), 113-140.

10. J. W. Cahn and J. E. Hilliard, "Free Energy of a Nonuniform System. I. Interfacial Free Energy," J Chem Phys, 28 (1958), 258267.

11. S. G. Kim, W. T. Kim, and T. Suzuki, "Phase-Field Model for Binary Alloys,” Phys Rev E, 60 (1999), 7186-7197.

12. J. Z. Zhu, T. Wang, A. J. Ardell, S. H. Zhou, Z. K. Liu, and L. Q. Chen, "Three-Dimensional Phase-Field Simulations of Coarsening Kinetics of $\gamma$ ' Particles in Binary Ni-Al Alloys,” Acta Mater, 52 (2004), 2837-2845.

13. T. Mura, Micromechanics of Defects in Solids, $2^{\text {nd }}$ Rev. Ed. (Dordrecht: Kluwer Academic Publishers, 1987), 1-15.

14. A. G. Khachaturyan, Theory of Structural Transformations in Solids (New York: Dover Publications, 2008), 199-212.

15. S. Y. Hu and L. Q. Chen, "A Phase-Field Model for Evolving Microstructures with Strong Elastic Inhomogeneity,” Acta Mater, 49 (2001), 1879-1890.

16. X. H. Guo, S. Q. Shi, and X. Q. Ma, "Elastoplastic Phase Field Model for Microstructure Evolution,” Appl Phys Lett, 87 (2005), 221910.
17. F. K. G. Odqvist, Mathematical Theory of Creep and Creep Rupture (London: Oxford University Press, 1966), 19-33.

18. Y. Terada, T. Yasui, and T. Matsuo, "Creep of [011]-Oriented Ni-20mass\%Cr Single Crystals,” Mater Sci Forum, 426-432 (2003), 785-790.

19. T. Tokumoto, Y. Terada, and T. Matsuo, "Low-Stress Creep of Ni-20mass\%Cr Single Crystals with [001] Orientation," Mater Sci Forum, 426-432 (2003), 803-808.

20. D. Siebörger, H. Knake, and U. Glatzel, "Temperature Dependence of the Elastic Moduli of the Nickel-Base Superalloy CMSX-4 and its Isolated Phases,” Mater Sci Eng A, 298 (2001), 26-33.

21. U. Glatzel, "Neutron Scattering Experiments with a Nickel Base Superalloy Part II: Analysis of Intensity Profiles," Scripta Metall Mater, 31 (1994), 291-296.

22. H. Izuno, T. Yokokawa, Y. Koizumi, S. Okada, and H. Harada, "A Creep Constructive Equation of Ni-Base Superalloys Using Structural Parameters,” J. Japan Inst. Metals, 68 (2004), 526-529.

23. Y. Tsukada, Y. Murata, and T. Koyama, "Phase-Field Simulation of Rafting Caused by Creep Deformation in Ni-Based Superalloys" (Accepted for Publication in the Proceedings of the $12^{\text {th }}$ International Conference on Creep and Fracture of Engineering Materials and Structure, Kyoto, Japan, 27-31 May 2012).

24. Y. U. Wang, Y. M. Jin, A. M. Cuitiño, and A. G. Khachaturyan, "Nanoscale Phase Field Microelasticity Theory of Dislocations: Model and 3D Simulations,” Acta Mater, 49 (2001), 1847-1857.

25. M. E. Kassner, Fundamentals of Creep in Metals and Alloys (Amsterdam: Elsevier, 2009), 247-259. 